'The guy might not even be able to remember why he's here and what he's in here for and why he's locked in': Residents and prison staff experiences of living and working alongside people with dementia who are serving prison sentences for a sexual offence 


\section{'The guy might not even be able to remember why he's here and what he's in here for and why he's locked in': Residents and prison staff experiences of living and working alongside people with dementia who are serving prison sentences for a sexual offence}

Prison staff and residents with sexual convictions, from two UK prisons, were interviewed to explore their understanding and experiences of dementia. The aim was to explore understanding and experiences of dementia in response to Governor-identified needs in the prisons. Semi-structured interviews were conducted with thirteen prisoners and seventeen prison staff across both prisons, all of whom had direct prisoner contact. Transcripts of the interviews were created and thematic analysis was carried out. Four superordinate themes emerged from the data. The first theme, 'Balancing act', explored the way that individuals with dementia should be encouraged to be independent whilst still meeting their need for support in some tasks and areas of functioning. The second theme, 'Challenges and confusion', explored the way that dementia symptoms might be masked in prison settings, how they can be misinterpreted and the impact and understanding of inappropriate behaviour in a prison for those with sexual convictions. 'What works and what doesn't' in a prison setting formed the basis of the third theme, with the final theme, 'Who to tell?' exploring the transfer of information and the sources of support available in the prisons. Practical suggestions for caring for individuals with dementia in prison are made.

Keywords: dementia; prisons; staff and prisoner experiences, vulnerable populations, sexual convictions 


\section{Introduction}

Dementia (also known as Neurocognitive Disorder; DSM-V, American Psychiatric Association, 2013) is a term given to a cluster of symptoms that are observed across a range of progressive organic neurodegenerative diseases of the brain, such as Alzheimer's disease, vascular dementia, dementia with Lewy bodies, fronto-temporal dementia and Parkinson's disease (APA, 2013). Symptoms of dementia typically include short-term memory problems as a core feature, alongside a decline in other areas of cognitive and executive function, communication difficulties, perception difficulties, mood symptoms, and personality changes (APA, 2013), which progressively become more severe and eventually lead to a loss of ability to live independently.

Dementia has become an increasingly prevalent challenge for contemporary health and social care providers, and this increased prevalence has largely been attributed to the increase in the number of elderly individuals in the general population (Prince et al., 2014). Age is a central factor in determining an individual's risk of developing dementia, with those aged 60 years old and above being more susceptible to developing the condition (Holmes \& Amin, 2016). The Alzheimer's Society (2014b) estimated that 815,827 people in the UK had dementia, and forecast that this figure would likely increase to $1,142,677$ by the year 2025 as a result of demographic aging (Alzheimer's Society, 2014b).

This increase of elderly people in the community has also been paralleled in prison populations, and the number of prisoners over the age of 60 has also grown (Moll, 2013; Nacro, 2009). It is suggested that approximately 5\% of prisoners aged 55+ have dementia, and statistics clearly show that corresponding with the ageing 
population, there is a steady rise in the number of elderly individuals convicted of a sexual offence (Bows \& Westmarland, 2018).

This ageing population is reflected in HMP Whatton and Stafford, where the average age of the men is the mid-40s. With the numbers of non-recent sex abuse convictions also increasing, this is likely to again be reflected in the population of men at Whatton and Stafford, thus highlighting the need to focus on the impact of dementia in men currently serving a custodial sentence.

The UK government's National Dementia Strategy (Department for Health, 2009) sets out key recommendations to help improve dementia care services across institutions such as prisons and the NHS. These include raising awareness and understanding, improving early diagnosis and support, and living well with dementia. The decency agenda in prisons can be used to support this strategy by ensuring that people with dementia feel valued, that they are assisted with things they find difficult (such as personal care) with dignity and by ensuring that the needs and sensitivities relating to dementia are understood within the prison.

The way that dementia is dealt with within a prison can have an impact on the wellbeing of the prisoner after release. Ginn (2012) suggests that because of the structure and routine of the prison regime, the early stages of dementia may go unrecognised. This can be harmful in life outside the prison as the loss of structure can magnify the difficulties an individual faces without the appropriate support.

What is more, research suggests that older prisoners may be at a higher risk of developing dementia compared to elderly individuals in the community (Christodoulou, 2012). It has been suggested that prisoners age, physiologically, at an accelerated rate in comparison with people living in the community. This acceleration of physiological age has been attributed to the nature of life in prison, which increases risk factors and 
provides fewer protective factors for dementia (Wilson \& Barboza, 2010). Some examples of these factors include a sedentary lifestyle coupled with poor nutrition, a higher prevalence of smoking amongst prisoners, prior to the prison smoking-ban, (Christodoulou, 2012), and an increase in general psychological strain of incarceration in a prison (Sterns, Lax, Sed, Keohane \& Sterns, 2008).

\section{Challenges faced by prisoners with dementia}

Dementia can make life extremely difficult for the individual diagnosed with the condition, particularly as it progresses into the more severe stages (White et al., 2017).

\section{Victimisation}

One challenge that may threaten the wellbeing of individuals with dementia is an increased risk of being victimised by fellow prisoners. Previous research has highlighted that older prisoners are more likely to feel anxious about the possibility of being bullied, in addition to reporting experiencing bullying. Davoren et al. (2015) found that $38 \%$ of older prisoners, in their sample, had a history of being victimised or bullied in the prison setting, compared to $12 \%$ of younger prisoners. For some older prisoners, having dementia may further increase their risk of becoming victimised; they may be recognised as easy targets for bullying, be less able to defend against physical and sexual attacks, and less physically and mentally resilient in the face of victimisation (Davoren et al., 2015).

\section{Social isolation and depression}

Another risk faced by prisoners with dementia is an increased propensity toward apathy and social withdrawal (Alzheimer's Society, 2017), particularly in the middle and later 
stages of the condition. This may be subtle and easily misinterpreted by others as an expected outcome of living in a prison and a consequence of old age (Moll, 2013), or may be mistaken for other mental health issues rather than indicating dementia, for example depression, given their similar, overlapping symptomology, and common comorbidity (Alzheimer's Society, 2017). Older prisoners more generally tend to pose fewer security threats and are typically stereotyped as being quiet (HM Chief Inspectorate of Prisons, 2004). Therefore, an individual with dementia, who becomes socially withdrawn, may be mistaken for a prisoner who is simply quiet and nondisruptive.

However, withdrawal from regular social interaction can be problematic for individuals with dementia. Positive social interaction has been found to be useful in preventing cognitive decline in elderly populations (Kim et al., 2016). Social interaction has been suggested to be helpful in managing difficulties commonly associated with dementia and can reduce the likelihood of behaviours which are deemed to be challenging by others (Alzheimer's Society, 2014a). Conversely, social isolation has also been associated with increased risk of developing, or worsening existing, depression (Alzheimer's Society, 2016).

\section{Confusion, distress, anxiety and agitation}

Episodes of confusion, and consequent distress and anxiety or agitation and aggression, are commonly observed in individuals with dementia, and may emerge as a result of experiencing memory loss, communication difficulties or disorientation (Alzheimer's Society, 2014a). In a prison environment, this may mean individuals struggle to navigate the prison and become lost and disorientated. As the dementia becomes more advanced, they may not even realise they are in a prison, or understand why, which 
raises ethical concerns with regard to keeping them detained in a prison establishment (Fazel, McMillan \& O’Donnell, 2002).

Individuals with dementia may become less able to recognise their needs, or how to meet them appropriately. This may lead to inappropriate behaviours, such as undressing when they are too hot and resultantly exposing themselves, or toileting behaviours in communal areas because they cannot access private facilities easily (Alzheimer's Society, 2014a). Additionally, sexual disinhibition and inappropriate sexual behaviours (ISBs), such as public masturbation, excessive touching of others and sexual aggressiveness have been increasingly reported as a behavioural feature of dementia (Canevelli et al., 2011), occurring in $2-17 \%$ of people with dementia (Series \& Dégano, 2005). ISBs have been said to pose challenges in residential care contexts, in particular difficulties for staff in distinguishing ISBs from normal sexual behaviour (De Medeiros, Rosenberg, Baker \& Onyike, 2008). Problematically, it is likely that in a prison setting such behaviours could also be interpreted as offence paralleling behaviour, if the prisoner already has a sexual conviction. Other problematic behavioural changes that are commonly observed in people with dementia include shouting or screaming, following people, restlessness, accusing, 'sundowning' (increased agitation, aggression or confusion in the evening), and hiding objects (Alzheimer's Society, 2014a). In a prison setting these behaviours may lead to being reprimanded for such things as being late to roll call, or confrontations with staff or other prisoners, which could further compromise their mental and physical well-being.

\section{Environment}

Features of the environment are important considerations (Alzheimer's Society, 2014a). Some environments can be over-stimulating for an individual with dementia; for 
example, they may experience a hypersensitivity to temperature changes or lighting (Alzheimer's Society, 2014a). Environments with competing sensory information can be distressing and disorientating. In a prison environment, this may take the form of heavily crowded and noisy landings or corridors, which may increase feelings of distress, confusion or agitation, and may increase their propensity toward demonstrating some of the problematic behaviours highlighted earlier, and possible subsequent reprimand.

As a consequence of the visuoperceptual difficulties commonly experienced by people with dementia, prisoners may have further difficulty navigating the prison or completing tasks of daily living. People with dementia can struggle to judge distances, have difficulty differentiating objects in environments with competing visual information, or similar colours, and can develop impairments to the visual field, with reduced peripheral vision (Alzheimer's Society, 2016). As a result, when attempting to navigate a prison environment, prisoners with dementia may accidentally bump into objects or people, try to avoid shiny floors, perceiving them to be slippery, or avoid stepping on shadows, because they may be perceived as a change of level (Alzheimer's Society, 2016). Similarly, difficulties with differentiating objects from backgrounds of similar colours can impact on mealtimes, for example identifying mashed potatoes on a white plate, or correctly judging distances when reaching for objects, such as handrails or drinks, resulting in spillages (Utton, 2009). This can be problematic as it has the potential to cause ridicule or embarrassment (Prizer \& Zimmerman, 2018).

\section{Challenges in identifying and managing dementia}

Dementia in a prison setting can also present challenges for others in the prison, particularly for the members of prison staff who are charged with managing and 
supporting prisoners daily, whilst also being expected to maintain security and the smooth operation of the establishment.

A key difficulty that members of prison staff may face is identifying prisoners who have dementia. Although it is not their role to make diagnoses, offering appropriate support and signposting to healthcare for screenings and diagnosis. In the community, referrals for dementia screenings generally occur as a result of family, friends or that individual's professional support network recognising their diminished ability to live independently, and many screening approaches focus on the capacity to complete instrumental tasks of daily living as a key feature of the assessment process (Feckzo, 2014). However, this may not be as straightforward in a prison setting. It has been posited that the heavily structured, regimented nature of most prisons can have a masking effect, making dementia more difficult to detect (Sterns et al., 2008). Many of the instrumental activities of daily living (Simmons, Hartmann \& Dejoseph, 2011) such as cooking, shopping and driving, are either completed for individuals in prisons or are not required (Williams et al., 2012), and their value in dementia screening is therefore obsolete (Feckzo, 2014). As a result, it may not be until the more severe stages of the condition that signs of dementia are recognised.

The paucity of dementia-specific training delivered to prison staff has been highlighted as a contributor to the identification problem (Williams et al., 2012). Without specific training of what to look out for, frontline prison staff may struggle to recognise signs of dementia, particularly in light of the masking effect of the regimented nature of prison (Sterns et al., 2008). 


\section{Challenges to managing dementia in a prison}

Delivering appropriate and adequate support and management strategies for prisoners with dementia can also be difficult. There are a number of restrictions in the prison environment which may make it difficult to provide the appropriate level of care to an individual with dementia, such as security, budget and resource constraints.

It has been proposed by some that a separate wing or landing for older prisoners with these conditions could be helpful, where they can receive specialised healthcare support. However, for many establishments, this requires a costly initial investment, and may, therefore, not be realistic (Williams et al., 2012). Other suggestions put forward include the idea that long-term costs would be reduced by centralising the older prisoner populations, making it easier to deliver appropriate care (Kerbs \& Jolley, 2009).

However, it has also been counter-argued that although a separate landing or wing may appear prima facie to be a useful idea, segregation from the wider prisoner community may have negative effects on the individual, and that inclusion and interaction with the wider prisoner social network, such as with younger prisoners, may be more beneficial for both them and their younger counterparts (Moll, 2013).

With the increased recognition of dementia as an increasingly prevalent health issue in the general population, it has become a key focus of much contemporary health and social care research, policy and practice. However, this has not extended, to the same degree, to prison settings, and there has been a call for further research. Moll (2013) highlighted that there has been a promising increase in research focussing on elderly prisoners generally, yet little attention has been focussed specifically on the issue of improving management and support for those with dementia in this population.

In the UK, the prison service has identified the emerging needs regarding the management, care and support for prisoners with dementia. This research was carried 
out in conjunction with two prisons, both category $\mathrm{C}$, housing adult males convicted of sexual offences (HMP Whatton and HMP Stafford). The aim of the research was to qualitatively explore understanding and experiences of dementia in both prisons through interviews with prisoners and members of prison staff. The research was undertaken in response to Governor-identified needs in the prison with the intention of informing recommendations for realistic and meaningful, change.

\section{Method}

\section{Sample}

Participants comprised thirteen adult male prisoners and one transgender (female) prisoner currently serving a sentence at a UK Category $\mathrm{C}$ prison housing people convicted of sexual offences. There were seventeen prison staff participants (ten female; seven male) from across two UK category $\mathrm{C}$ prisons, all of whom had direct prisoner contact. Staff participants included prison security officers of various ranks, volunteers, and members of the prison healthcare team.

\section{Ethics}

Ethical approval was obtained from Her Majesty’s Prison and Probation Service National Research Committee and Nottingham Trent University (NTU) College Research Ethics Committee prior to commencement.

\section{Procedure}

Prisoner participants were approached through the circulation of information packs across four wings of the prison that housed a concentration of older prisoners. 
Information packs were also distributed to prisoners who attended a presentation about dementia, delivered to the Older Prisoner's Activities and Learning (OPAL) group, by the research team. Prison staff participants were recruited by circulating information about the study via a global staff email in one prison and through a deputy governor at the second prison.

Participation was voluntary and conferred no additional external benefit to participants. Individuals who were interested in participating were asked to contact the research team to arrange an interview. The majority of the participants noted that they had personal experience of dementia both internal and external to prison settings, which underpinned some of their reasons for taking part in the study. There were few participants who had not had any prior experience or understanding of dementia.

\section{Data collection}

Interviews were conducted on a face-to-face basis. Interviews lasted between 10 and 87 minutes ( $M=31$ minutes), were recorded on a passcode-protected dictaphone and transcribed using a primarily secretarial style of transcription. Names and places were anonymised; where names are used in quotations, they are pseudonyms.

Interviews with prisoners were conducted in private interview rooms, or the prison's Older Persons Assessment and Liaison Team cabin. Interviews with staff were conducted in private offices or psychology interview rooms.

The interview schedule was developed from existing literature, with additional input from prison psychologists; it encouraged participants to reflect on their knowledge and experience of dementia in prison and/or in the community. The schedule comprised a number of broad sections including questions regarding the potential impact of dementia on daily life and everyday activities within the prison context, what additional 
challenges (and benefits) individuals with dementia might experience living in the prison environment, together with reflections on any adaptations or processes that might prove beneficial to individuals with dementia in prison, or the staff or residents who supported such individuals.

\section{Mode of analysis}

Interview transcripts were analysed by the research team using thematic analysis (Braun \& Clarke, 2006). Thematic analysis was chosen as a flexible yet systematic method of qualitative analysis that allowed researchers to assemble themes that emerged inductively from the interview data. Since the aim of this research was to contribute in a practical manner to efforts by prisons to improve the quality of life of prisoners with dementia, a form of analysis we have termed applied inductive thematic analysis was completed. The goal of applied inductive thematic analysis is to describe and understand how people feel, think, and behave in a particular context in relation to a specific real-world research question, in as rigorous and meaningful way as possible (Guest, MacQueen, \& Namey, 2011). It is particularly appropriate in applied contexts, in response to a practical need for guidance (in this case, two prison governors requesting help in improving the quality of life for their prisoners with dementia, and the staff and prisoners working and supporting such individuals). The analysis is inductive in that the findings and themes were allowed to emerge freely from the data, rather than being driven by prior theoretical or practical assumptions.

Authors of the current study familiarised themselves with the transcripts; this involved reading, re-reading, note taking, developing initial clusters of themes for each transcript and grouping them together across the accounts. The authors maintained a specific focus on extracting applied themes. During the coding process authors 
independently extracted codes from the transcripts to establish subordinate themes. The authors collaborated and discussed their interpretations of the data to establish commonalities in codes and primary themes. Several iterative stages of analysis and theming took place, with a constant re-checking and re-grounding back to the transcripts, before the final thematic structure was agreed by the researchers. This iterative process of cross-checking (between researchers) and re-checking (between emergent themes and transcript data) helps to underpin reliability in qualitative research, echoing the techniques of inter-rater reliability and test-retest reliability in quantitative research methods.

\section{Results}

Four superordinate themes emerged from the rich data set; these are detailed in Table 1 with respective subordinate themes.

Table 1 about here

\section{Superordinate theme 1: Balancing act}

Many participants emphasised that supporting prisoners with dementia required a balancing act by prisons, prison staff and other prison residents. Too much help or specialist services could lead to the further isolation of individuals; however, a lack of awareness, or failure to be responsive to the needs of a prisoner with dementia, could be harmful in a plethora of ways. 
Dementia is characterised by a gradual loss of ability to live and function independently, particularly in relation to the completion of daily activities, such as eating, dressing, toileting and maintaining personal hygiene (Gove et al., 2017). Participants in this study acknowledged the difficulties they faced living and working alongside prisoners with dementia, as they tried to provide the additional daily help necessary for these individuals to complete their daily activities, whilst also trying to leave the individual with dementia with a sense of independence and personal autonomy.

'Well the er er basically it er I mean everybody helps him with er, doing his menus and everything like that, and, I say basically it it's down to, help with paperwork and things like that, \{well it's obvious\} it wouldn't get done otherwise.' Prisoner 6 'Erm, notes up on the wall, er, cause I know [Prisoner with Dementia] forgets where his toilet is, and its only there, so to have notes on different things, times, erm and very often in large print, so that they can see it.'

Participants gave specific examples of ways that they have, or could, support prisoners with dementia.

$P$ : 'In the navy sort of thing like, morning sir and really you know erm, but I've seen guys, I think because of how my mum is, you look round and can see some of the older guys and think this guy's, perhaps got a problem with with dementia, er, you'll you perhaps see a guy that's lost erm can you tell me where this is so it's like come on I'll if you point them in that direction they're going to get lost again 


\section{R: Yeah}

P: So take take them and and walk round to where they're supposed to be sort of thing'

Many participants emphasised that support should be measured and proportionate to the severity of a person's dementia. Participants argued that independence and the loss or maintenance of that independence is a central difficulty faced by those with dementia. Therefore, it is important to nourish any capacities of independence where they exist. This may include adjusting the way in which support is provided, rather than simply providing it or not providing it- an example of this was given by one participant, in relation to navigating the prison. It was suggested that this could also include measures often seen and recommended in community settings, for example the use of clearer signposting or highly contrasting coloured lines on the floor to key locations within the prison, such as healthcare. This type of support can be helpful for everyone (not just prisoners with dementia) and as it constitutes a generic change to the prison environment does not focus on people with dementia but supports them in navigating the prison independently.

\section{Subordinate theme 1.2: Specialist treatment versus special treatment}

Participants also highlighted that if a prisoner does have dementia, staff should consider the possibility that dementia may be a contributing factor to their behaviour when enforcing prison rules. Particular behaviour may be dementia-related rather than a deliberate violation of rules. For example, is a person somewhere in the prison that they should not be because they have wandered and become lost, or is it a deliberate violation of the rules? 
'And so we have a robust system in place, whereas when people are a little bit confused there needs to be erm, we have to cut them some slack we have to work with the condition, and, yes they are a prisoner, but there has to be a fair amount of understanding that actually their thought processes are not the same as another prisoners and of course that's a big issue'

It was emphasised that staff should reflect on this possibility, taking dementia into consideration. However, there may also be occasions where, despite the wrongful behaviour being probably due to dementia, for risk and security reasons an adjudication may still be appropriate, both to emphasise that the behaviour was wrong and to protect others.

A second point within this theme was the question of whether there was a need for specialist wings or landings for prisoners with dementia, to ensure their care and support needs can be met. However, this must be considered in balance with the benefits of social interactions facilitated by being on standard wings.

'I'm just thinking aloud, would a dedicated landing for people with dementia, help, so that's like er, you know because other prisoners that's what you've got to think of when a guys, if they walked into someone else cell, my mums walked into other people's rooms, what are you doing in here, are you pinching my stuff sort of thing, and the prisoners won't understand what dementia is.' $\quad$ Staff 4 'they are all mixed ages which is right, it's the best way to be, and a lot of our young ones will help the older population, and it works well within that' Staff 13

\section{Superordinate theme 2: Challenges and confusion}

Participants highlighted several challenges when considering dementia with prisoners. 
Subordinate theme 2.1: Masking of dementia

Certain features of prison life mask dementia; in the community, dementia is often identified when a person struggles to carry out day to day activities that allow them to live independently. The structure and routine of a prison regime can support the individual by offering familiarity and removing some of the tasks associated with independent living. However, such structure may also mean that it is more difficult to identify initial stages of dementia in prisoners.

'his prison life hangs on those routines, he knows this is happening this date this time and that's really, been really useful to him... it's helped him be n-, be normal... in inverted commas...erm where he doesn't constantly have to go and ask people what's happening, where am I supposed to be, what am I supposed to be doing erm and things like he comes here and I play domi-, well a few of us play dominoes'

'Prisoners with dementia, erm, recognition, first and foremost, erm, how do you recognise, how do they recognise they've got dementia, erm (.) like I said, it's an insidious illness, it doesn't *clap* automatically, it just creeps up and creeps up and you're not kind of aware that you know, you have a problem or an issue, and I think that's the diagnosis of it, is the difficult thing, especially in this environment...' 
'Because it's so regimed, the routine is here, and if they've been completely fine, and then the dementia's crept in while they've been here you normally find you don't see many problems because they'll just carry on with the routine' Staff 1

\section{Subordinate theme 2.2: Misattribution of symptoms}

Participants highlighted that dementia could be mistaken for intellectual disability, particularly as some features could appear similar e.g. confusion or memory difficulties. Similarly, those with dementia may be wrongly assumed to have depression, demonstrating similar behaviour to someone who is depressed: being quiet and socially withdrawn. Family and friends who visit a prisoner with dementia may assume that their increasing social withdrawal is a consequence of their time in prison, or lack of interest in the visit, rather than recognising possible dementia. Encouraging families to maintain connections with a family member in prison could assist prison staff with making sense of changes in behaviour, and the Farmer review (2017) detailed the importance of sustaining family connections for wellbeing and reducing reoffending. 'I think that was something I didn't understand when I first started, because somebody had been really quite volatile, and I hadn't known anything at all about dementia, and I was on with another officer who had, who was from another jail, and she was like yes my mother has it, and she's just like that, ... and I was thinking oh ok, that's interesting, having somebody telling you that helps, he more we can promote that information, and perhaps change the mindset of people who assume, that this is deliberate behaviour, then I think it will help' 
Subordinate theme 2.3: Inappropriate behaviour

Prisoners with dementia may act inappropriately, with outbursts of anger, inappropriate sexual behaviour and poor hygiene/toileting. These dementia-related behaviours may be assumed to be deliberately inappropriate behaviours, particularly if a member of staff is unaware of the person's dementia.

'if a guy with dementia comes onto the landing for example wearing his slippers he shouldn't be shouted at for not for you know cos in our wing you're not allowed to wear your slippers outside of your cell'

\section{Prisoner 16}

'Some might see it as odd behaviour, it could be, could be construed as noncompliance, it could, it could become an issue if it wasn't picked up on, or someone not wanting to engage in the regime, it could be seen as laziness, erm, so, yeah, some people are better at picking up on things' Staff 9

\section{Superordinate theme 3: What works, what doesn't work}

In this theme, participants highlighted what, in their experience, seemed to be helpful or unhelpful in supporting prisoners with dementia.

\section{Subordinate theme 3.1: Interacting with others}

Prisoners with dementia typically present as socially withdrawn; they may not come to the notice of staff since they are well behaved and do not present as a threat to security. Regular social interaction and activities were identified as important in supporting individuals with dementia, particularly in the prison environment where it could become easy to slip into a self-isolating daily routine. Social activities, such as those provided 
by OPAL, together with the convivial community spirit on wings were emphasised as extremely important to help support prisoners with dementia.

'Well I think, they tend to become more insular. They tend to become more withdrawn. They don't want to mix in. They erm, have a fear of opening up to people' Prisoner 3

'It is important to just come up with little things that would include them, I mean, this chap, he likes his Irish drum, so we're going to get him to teach other people to play the Irish drum, so he can be, a part of the group in that way, have music and a sing along, and be able to put something in, and, and feel appreciated, which obviously will help him'

\section{Subordinate theme 3.2: Learning from the mainstream}

Participants highlighted a number of community initiatives that could work well in the prison environment, some of which were based on their own experiences of dementia in the community. These included the introduction of high contrast colours in the environment (e.g. red toilet seats and bedding), clear signage to aid navigation of the prison (e.g. coloured lines or footprints to key locations such as healthcare), large posters with meal choices and familiar pictures of themselves on cell doors were all given as examples of simple but useful adjustments that could be made to the prison environment. All of these would help prisoners with dementia maintain some independence and reduce stress arising from difficulties navigating daily life.

'well, obviously there is a lot of, coming from a nursing home, there is a lot of activities, all the activities are based around reminiscing, and stuff like that, which would probably be nice to do in this environment as well' 
'I mean, some of the other younger guys, Euan, his buddy is 23, and he, he, I think he cares for his parents on the outside, he enjoys it as well, it's just picking the right people for the right job'

Staff 8

An example of what may not work in prison however came from some techniques that are used in standard dementia practice in the community. Techniques that are used in the community to support those with dementia could be useful for prisoners but may need to be adapted. For example, memory life stories and music therapy projects such as Music Works (Osborne, 2015) which uses music to build wellbeing and support memory are a common technique in community dementia practice, but it could be potentially problematic or traumatic for an inmate to have to reflect on their whole past, so a reflection on positive life moments may be more appropriate. Equally, treatment approaches for offending behaviour may also need adapting for those with dementia.

\section{Superordinate theme 4: Who to tell?}

The issue of who should know about an individual's dementia diagnosis was raised as an important concern. Participants were mindful of people's need for privacy, set against staff (and prisoners) finding it useful to know so they could better support these individuals. The issue of an individual receiving a diagnosis, and what this meant for them, was also highlighted. It may also be useful to encourage the disclosure of dementia to family members and external friends. This may help widen and augment the support network for that prisoner, but also could be helpful for visits and forward planning for challenges that individual may face post-release. 
Subordinate theme 4.1: Screening, diagnosis and care

Currently in the UK, prisons do not typically screen for dementia. However, in a small number of cases (or perhaps where the dementia is quite advanced) a formal diagnosis may have been made. Participants suggested that a formalised approach to screening for dementia (with accessible information about how to make a referral) would be helpful, so that staff could refer prisoners and prisoners could self-refer.

'it's a collective thing between healthcare, and I think they need to pull their socks up a little bit more and have er more erm examinations of the elderly er irrespective of their age, you know, I don't know when you want to, when they should start the age, but from what I've heard there's been young people well under 60 that have suffered with dementia'

Participants also discussed who should know about the diagnosis, with suggestions that wing officers and external escorts should be informed to ensure that they can provide adequate support and apply appropriate management strategies both in the prison and on external hospital visits. Ongoing training and education on identifying dementia for staff and prisoners were also suggested as being helpful in identifying and supporting prisoners who are potentially demonstrating signs of dementia.

'I've never seen anything on an escort about dementia taking people out to hospital, there's no, certainly no, guides certainly nothing for staff to, to explain to people what is happening just turn up and drag them off take them to the 
hospital without any sort of explanation of what we're doing so, some, you know people are diagnosed'

A central issue highlighted by participants in the present study was the need for improved interdepartmental communication within the prison, and a more collaborative, multidisciplinary approach to the support and management of prisoners with dementia, or suspected dementia. Suggestions included improved communication and collaboration between wing officers and healthcare staff, and clearer protocols for reporting important information between departments, such as reporting officer observations of concerning dementia-indicative behaviours to the healthcare department.

'Because she said it wasn't right that our personal history should be available to anybody in the prison like that, especially our medical history, so unfortunately the scheme got scrapped'

Prisoner 4

In this extract, prisoner participant four is reflecting on the difficulties faced by prison staff of balancing the need for confidentiality with being able to know and support prisoners with dementia diagnoses. Previous literature has also brought this issue to light, reinforcing the suggestion that a well-coordinated system of information sharing between relevant staff groups and agencies, and between different prisons, is conducive to supporting prisoners with dementia (Moll, 2013).

\section{Subordinate theme 4.2: Living with a label}

This subtheme follows on from the notion of offering a referral and diagnosis service for prisoners. Participants asserted that this should be introduced sensitively, to avoid 
generating fear of dementia amongst older prisoners. The self-construal of individuals with a dementia diagnosis should also be considered when communicating messages about dementia within the prison. On the other hand, not having a label could attract name calling:

'but if someone's like, 'oh erm, why is he talking to himself?' or 'why is he talking to a window?' 'There's no-one there. What a thicko. What a weirdo!' You know what I mean? Nobody would know' Prisoner 10

This quote highlights the importance of a prison-wide understanding of dementia, to avoid stigma and negative interactions with others. Labels and diagnoses can be useful to helping those working with or living alongside someone with dementia to understand behaviours that might otherwise be misunderstood, although they should be introduced sensitively to avoid promoting anxiety in the person living with dementia.

\section{Subordinate Theme 4.3: Supportive others}

Participants were vocal about the level of support for prisoners with potential dementia (or who needed help) from staff and peers on the wing, highlighting how beneficial this community spirit was for those with dementia.

'They're sat, not talk-, you know, lost in their own little world and I don't want to interfere, don't want to go, you know, but I have tried sitting next to someone who has been quiet for a long period of time... I realised that everybody that has their, their demeanour might change, depending on circumstances... so you have to be, so that the middle try and make people understand that you understand them... you know and that's why you ask me about people here, you 
know, I keep my eyes open as far as I'm able to and then if anybody wants to open or talk to me...'

'I think the social aspect is quite important, they like to mix with people who are the same age as them'

Participants suggested some of this support could be formalised by, for example, creating a dementia-buddy role, which could work alongside other dementia-specific support structures. This has also been reported in existing research as a recommendation, and a useful support strategy for prisoners with dementia that has been successfully implemented in other prisons (Hodel \& Sánchez, 2013; Maschi, Kwak, Ko \& Morrissey, 2012). 'yeah so erm you know we'd help them getting their food because they could forget to get their food erm you know just sort of general light sort of cell cleaning on a weekend to help'

Prisoner 1

\section{Discussion}

The aim of the current study was to explore experiences and understanding of dementia in a prison setting, from the perspective of individuals who live and work in two UK prisons. More specifically, given the dearth of existing research exploring experiences and understanding of dementia in prison settings, this research sought to identify the current issues posed by dementia in a prison environment and the provisions that were available within the prison to support and manage prisoners with dementia. Moreover, there was an overarching aim to work towards developing practical recommendations to improve these support and management provisions, within UK prisons. 
A number of themes were identified from the transcripts: the balancing act of encouraging autonomy and providing sufficient support to someone with dementia; challenges around the identification of dementia related behaviours; finding appropriate methods of support and identifying what is not working well; and making decisions about how a dementia label and support needs are communicated. These issues, and subsequent recommendations, stemming from this analysis and existing literature, are outlined below.

\section{Identifying dementia in prisoners}

Recognising signs of dementia in prisoners was identified as a core challenge by participants in this study, and the introduction of effective early screening strategies was stressed as a priority area, in order to implement and direct appropriate support and management strategies. Participant emphasis on early screening strategies, which was found in this study, is consistent with mainstream community-based literature on dementia, which suggests that referral to specialist teams in the initial stages of the condition is vital for early diagnosis and useful intervention strategies (Holmes \& Amin, 2016).

Both in this study and in previous research, routine dementia screenings for elderly prisoners, for example annual screenings for prisoners over the age of 55 , has been advocated (Williams, Stern, Mellow, Safer \& Greifinger, 2012). To aid with the challenge of recognising dementia in residents, it has been proposed that 'prison based functional impairment' should be defined (Williams et al., 2012), for example, is a resident capable of completing 'Prison Activities of Daily Living' (PADLs) (Williams et al., 2006; Feckzo, 2014) independently? If frontline staff, such as wing staff, notice a 
resident having consistent difficulties in completing PADLs independently, then this may be a useful prompt for them to refer a resident for a dementia screening.

\section{Central importance of increased awareness and training}

The need for an increase in awareness of dementia in the prison was another salient issue in this study, with participants suggesting that an increase in the availability and accessibility of dementia-specific awareness and training events in the prison could be useful. It was suggested that a broader awareness of dementia in the prison could contribute toward generating a climate where support is more accessible and readily available for residents with dementia.

A sense of community spirit in the prison was emphasised by participants in this research, with operational staff seeking to take on a more supportive role and residents willing to support fellow inmates. With increased awareness and understanding of dementia, individuals in the prison, staff and residents alike, may feel more able to provide this support, and more able to recognise signs of potential dementia.

This, again, is consistent with findings and priorities that have been put forward in previous studies, which have emphasised that specialised dementia training, delivered in prisons, could improve recognition of dementia symptomology and support for those with the condition (Maschi, Kwak, Ko \& Morrissey, 2012). Additionally, issues surrounding appropriately managing dementia-related behaviours may become mitigated as individuals learn to distinguish dementia-related behaviours from more general intentionally inappropriate or disobedient behaviours (Maschi, Kwak, Ko \& Morrissey, 2012; Williams, Stern, Mellow, Safer \& Greifinger, 2012). Such training could be cost-effectively delivered through the involvement of specialist external voluntary agencies (Moll, 2013). 


\section{The physical environment}

Many participants in this study suggested that the physical environment of a prison may not be suitable for individuals with dementia, and that the introduction of certain simple modifications to the physical environment could be useful for residents who have dementia. Participants drew from their experiences both in a prison context and experiences with their own family members with dementia in the community, that such modifications could promote independence for residents with dementia and mitigate feelings of confusion and stress, alleviating the impact of dementia upon both the individual and those around them.

This has also been a popular suggestion in previous mainstream and forensic literature, which postulates that certain alterations to the physical environment can serve to maximise independent functioning and compensate for the difficulties caused by dementia. These alterations can mitigate confusion, anxiety and agitation in individuals with dementia, by reducing the number of potential triggers of unwanted dementiaassociated behaviours (Utton, 2009).

In the current study, examples of modifications that were suggested by participants, and have been suggested in previous research include: clear signposting (with large letters and relevant pictures); sharp contrasting colour walls to indicate toilet facilities, to prevent incontinence arising from forgetting where a bathroom is; and large calendars that indicate routine events in the prison (such as cell cleaning day), all of which have been implemented in a number of prisons with positive outcomes (Moll, 2013; Hodel \& Sánchez, 2013; Maschi, Kwak, Ko \& Morrissey, 2012). These types of changes were highlighted by both staff and prisoner participants in the present study as relatively low-cost, as their relative simplicity means that current resident-workers 
could implement them. For example, resident maintenance teams could paint coloured lines or footprints that would become helpful for individuals with dementia to navigate to key locations in the prison, such as the healthcare department.

\section{Ethical \& legal implications of dementia in prison}

The analysis here raised some important issues to consider regarding the management and support of prisoners with dementia. Whilst there are a number of accommodations that could be made to improve the management and support for prisoners with dementia, it must be emphasised that they do not represent a permanent care strategy. In the early stages of dementia, when symptomology is subtler, certain accommodations can be made to appropriately support and manage those with the condition in a prison. However, in the latter, more complex, stages, it is important to acknowledge that the necessary specialist care requirements may well be beyond the remit and resources of a prison setting.

Furthermore, this raises a debate that has been previously highlighted in the literature, which question whether detaining an individual with dementia in a prison setting is ethically or legally defensible (Davoren et al., 2015). Moreover, Fazel, McMillan and O'Donnell (2002) argued that it is unlikely that the typical purposes of prison (deterrence, incapacitation, rehabilitation, retributive justice) would be appropriately applicable to those with dementia. For example, as the condition progresses to the more severe stages, individuals with dementia may become incapable of reflecting on their past offending behaviours, may become incapable of committing further offences, or may not even be aware they are in prison, or why they are there.

This argument further reinforces views expressed by participants in this study, who emphasised the unsuitability of, and argued against, detaining men with dementia 
in an environment that is not designed or resourced to adequately to meet their support and management needs.

\section{Considerations}

This research was intended to support the development of an older persons' strategy in two specific prisons in response to a Governor-identified need. The extent to which the findings may be applicable to other prison settings should be considered. The two prisons involved in this study cater for men who have been convicted of a sexual offence. In a previous section of this paper it was highlighted that the mean age of the prison population for these offences is older than in the rest of the prison estate. Prisons which focus on working with those with sexual convictions are also reputed to have a generally calmer environment, with fewer reported acts of violence than other types of prisons (Levins \& Crewe, 2015).

\section{Future Research}

Issues surrounding post-release resettlement for prisoners with dementia remain a challenge. Finding suitable accommodation for a recently released prisoner, who has sexually offended, can be a challenging task more generally (Evans \& Porter, 2015). When this is combined with the support services that an individual who has dementia requires, the difficulties of resettlement are compounded. Further research into the challenges of finding accommodation post release for individuals with specific care needs such as dementia who also have been convicted of a sexual offence should be a priority. 


\section{Conclusion}

This research has highlighted important issues that should be considered in relation to caring for individuals with dementia in a prison environment. Recommendations include having a strong emphasis on the importance and value of greater education, training and promotion of awareness of dementia, among both prison staff and prisoners themselves and making practical and affordable changes to the physical environment. 


\section{References}

Age UK (September, 2016) Caring for someone with dementia. Retrieved 28 June 2017 from http://www.ageuk.org.uk/Documents/EN-GB/Informationguides/AgeUKIG47_caring_for_someone_with_dementia_inf.pdf?dtrk=true

Alzheimer's Society (2014a) Changes in behaviour, Factsheet 525, Retrieved 10 August, 2017, from https://www.alzheimers.org.uk/download/downloads/id/3354/changes_in_behaviour.pdf

Alzheimer's Society (2014b). Dementia UK: Update. November, 2014. Retrieved 28 June, 2017, from https://www.alzheimers.org.uk/download/downloads/id/2323/dementia_uk_update.pdf

Alzheimer's Society (2016) Sight, perception and hallucinations in dementia, Factsheet 527, Retrieved 10 August, 2017, from

https://www.alzheimers.org.uk/download/downloads/id/3369/sight_perception_and_hall ucinations_in_dementia.pdf

Alzheimer's Society (2017) Apathy, Depression and Anxiety, Factsheet 444, Retrieved 10 August, 2017, from

https://www.alzheimers.org.uk/download/downloads/id/1768/factsheet_depression_and _anxiety.pdf

American Psychiatric Association. (2013). Diagnostic and statistical manual of mental 
disorders (5th ed.). Arlington, VA: American Psychiatric Publishing

Bows, H., \& Westmarland, N. (2018). Older sex offenders-managing risk in the community from a policing perspective. Policing and Society, 28(1), 62-74.

Braun, V., \& Clarke, V. (2006). Using thematic analysis in psychology. Qualitative research in psychology, 3(2), 77-101.

Canevelli, M., Troili, F., Talarico, G., Tosto, G., Letteri, F., Lenzi, G. L., \& Bruno, G. (2011). Inappropriate sexual behaviors in dementia: a review of literature. Cognitive Sciences, 6(2), 183.

Christodoulou, M. (2012). Locked up and at risk of dementia. The Lancet Neurology, 11(9), 750.

Davoren, M., Fitzpatrick, M., Caddow, F., Caddow, M., O’Neill, C., O’Neill, H., \& Kennedy, H. G. (2015). Older men and older women remand prisoners: mental illness, physical illness, offending patterns and needs. International psychogeriatrics, 27(5), 747-755.

Dawes, J. (2009). Ageing prisoners: Issues for social work. Australian Social Work, 62(2), 258-271. 
De Medeiros, K., Rosenberg, P. B., Baker, A. S., \& Onyike, C. U. (2008). Improper sexual behaviors in elders with dementia living in residential care. Dementia and geriatric cognitive disorders, 26(4), 370-377.

Department of Health (2009). Living well with dementia: A National Dementia Strategy. London: DH Publications.

Evans, D. N., \& Porter, J. R. (2015). Criminal history and landlord rental decisions: A New York quasi-experimental study. Journal of Experimental Criminology, 11(1), 21 42.

Farmer, L. (2017). The Importance of Strengthening Prisoners' Family Ties to Prevent Reoffending and Reduce Intergenerational Crime. Ministry of Justice.

Fazel, S., McMillan, J., \& O'Donnell, I. (2002). Dementia in prison: ethical and legal implications. Journal of medical ethics, 28(3), 156-159.

Feczko, A. (2014). Dementia in the incarcerated elderly adult: Innovative solutions to promote quality care. Journal of the American Association of Nurse Practitioners, 26(12), 640-648.

Ginn, S., 2012. Elderly prisoners. BMJ : British Medical Journal (Online), 345.

Gove, D., Scerri, A., Georges, J., Houten, P., Huige, N., Hayder-Beichel, D., Leichsenring, K., \& Morris, V. C. (2017). Continence care for people with dementia 
living at home in Europe: a review of literature with a focus on problems and challenges. Journal of clinical nursing, 26(3-4), 356-365.

Guest, G., MacQueen, K. M., \& Namey, E. E. (2011). Applied thematic analysis. London: Sage.

HM Chief Inspector of Prisons (2004), “"No problems - old and quiet”: Older prisoners in England and Wales'. Retrieved 10 July 2017 from https://www.justiceinspectorates.gov.uk/hmiprisons/wpcontent/uploads/sites/4/2014/08/OlderPrisoners-2004.pdf

Hodel, B., \& Sánchez, H. G. (2013). The Special Needs Program for Inmate-Patients with Dementia (SNPID): A psychosocial program provided in the prison system. Dementia, 12(5), 654-660.

Holmes, C, \& Amin, J. (2016). Dementia. Medicine, 44(11), 687-690.

Levins, A., \& Crewe, B. (2015). 'Nobody's better than you, nobody's worse than you': Moral community among prisoners convicted of sexual offences. Punishment \& Society, 17(4), 482-501.

Kerbs, J. J., \& Jolley, J. M. (2009). A commentary on age segregation for older prisoners: Philosophical and pragmatic considerations for correctional systems. Criminal Justice Review, 34(1), 119-139. 
Kim, C., Wu, B., Tanaka, E., Watanabe, T., Watanabe, K., Chen, W., ... \& Anme, T. (2016). Association between a Change in Social Interaction and Dementia among Elderly People. International Journal of Gerontology, 10(2), 76-80.

Maschi, T., Kwak, J., Ko, E., \& Morrissey, M. B. (2012). Forget me not: Dementia in prison. The Gerontologist, 52(4), 441-451.

Moll, A. (2013). Losing track of time. London: Mental Health Foundation.

Nacro (2009), 'A resource pack for working with older prisoners', Department of Health, GR 12279, London

Osborne, J. (2015). Soundtrack to My Life. JoCo.

Prince, M, Knapp, M, Guerchet, M, McCrone, P, Prina, M, Comas-Herrera, A, Wittenberg, R, Adelaja, B, Hu, B, King, D, Rehill, A \& Salimkumar, D. (2014). Dementia UK: Update. Second Edition. London: Alzheimers Society

Prizer, L. P., \& Zimmerman, S. (2018). Progressive Support for Activities of Daily Living for Persons Living With Dementia., The Gerontologist, 58(1), 74-87.

Series, H., \& Dégano, P. (2005). Hypersexuality in dementia. Advances in Psychiatric Treatment, 11(6), 424-431. 
Simmons, B. B., Hartmann, B., \& Dejoseph, D. (2011). Evaluation of suspected dementia. ratio, $1(5), 9$.

Sterns, A. A., Lax, G., Sed, C., Keohane, P., \& Sterns, R. S. (2008). The growing wave of older prisoners: A national survey of older prisoner health, mental health, and programming. Corrections Today, 70(4), 70-76.

United Nations, Department of Economic and Social Affairs, Population Division (2013). World Population Ageing 2013. ST/ESA/SER.A/348.

Utton, D. (2009). The design of housing for people with dementia. Journal of Care Services Management, 3(4), 380-390.

White, P. C., Wyatt, J., Chalfont, G., Bland, J. M., Neale, C., Trepel, D., \& Graham, H. (2017). Exposure to nature gardens has time-dependent associations with mood improvements for people with mid-and late-stage dementia: Innovative practice. Dementia, 1471301217723772.

Williams, B. A., Lindquist, K., Sudore, R. L., Strupp, H. M., Willmott, D. J., \& Walter, L. C. (2006). Being old and doing time: Functional impairment and adverse experiences of geriatric female prisoners. Journal of the American Geriatrics Society, 54(4), 702707. 
Williams, B. A., Stern, M. F., Mellow, J., Safer, M., \& Greifinger, R. B. (2012). Aging in correctional custody: setting a policy agenda for older prisoner health care. American journal of public health, 102(8), 1475-1481.

Wilson, J., \& Barboza, S. (2010). The looming challenge of dementia in prisons.

Correct Care, 24(2), 10-13. Retrieved 28 June, 2017, from

http://www.ncchc.org/filebin/images/Website_PDFs/24-2.pdf 